\title{
Targeting glutamine metabolism and the focal adhesion kinase additively inhibits the mammalian target of the rapamycin pathway in spheroid cancer stem-like properties of ovarian clear cell carcinoma in vitro
}

\author{
MASAKAZU SATO $^{1}$, KEI KAWANA ${ }^{1,2}$, KATSUYUKI ADACHI ${ }^{1}$, ASAHA FUJIMOTO ${ }^{1}$, MITSUYO YOSHIDA $^{1}$, \\ HIROE NAKAMURA $^{1}$, HARUKA NISHIDA ${ }^{1}$, TOMOKO INOUE ${ }^{1}$, AYUMI TAGUCHI ${ }^{1}$, JURI OGISHIMA ${ }^{1}$, \\ SATOKO EGUCHI $^{1}$, AKI YAMASHITA ${ }^{1}$, KENSUKE TOMIO ${ }^{1}$, OSAMU WADA-HIRAIKE ${ }^{1}$, \\ KATSUTOSHI ODA ${ }^{1}$, TAKESHI NAGAMATSU ${ }^{1}$, YUTAKA OSUGA ${ }^{1}$ and TOMOYUKI FUJII ${ }^{1}$ \\ ${ }^{1}$ Department of Obstetrics and Gynecology, Graduate School of Medicine, The University of Tokyo, \\ Bunkyo-ku, Tokyo 113-8655; ${ }^{2}$ Department of Obstetrics and Gynecology, \\ School of Medicine, Nihon University, Itabashi-ku, Tokyo 173-8610, Japan
}

Received January 10, 2017; Accepted February 17, 2017

DOI: 10.3892/ijo.2017.3891

\begin{abstract}
Ovarian cancer is one of the leading causes of death in the world, which is linked to its resistance to chemotherapy. Strategies to overcome chemoresistance have been keenly investigated. Culturing cancer cells in suspension, which results in formation of spheroids, is a more accurate reflection of clinical cancer behavior in vitro than conventional adherent cultures. By performing RNA-seq analysis, we found that the focal adhesion pathway was essential in spheroids. The phosphorylation of focal adhesion kinase (FAK) was increased in spheroids compared to adherent cells, and inhibition of FAK in spheroids resulted in inhibition of the downstream mammalian target of the rapamycin (mTOR) pathway in ovarian clear cell carcinomas. This result also suggested that only using a FAK inhibitor might have limitations because the phosphorylation level of FAK could not be reduced to the level in adherent cells, and it appeared that some combination therapies might be necessary. We previously reported that glutamine and glutamate concentrations were higher in spheroids than adherent cells, and we investigated a synergistic effect targeting glutamine metabolism with FAK inhibition on the mTOR pathway. The combination of AOA, a pan-transaminase inhibitor, and PF 573228, a FAK inhibitor, additively inhibited the mTOR pathway in spheroids from ovarian clear cell carcinomas. Our in vitro study proposed a rationale for the positive and negative
\end{abstract}

Correspondence to: Professor Kei Kawana, Department of Obstetrics and Gynecology, School of Medicine, Nihon University, 30-1 Oyaguchi-kamicho, Itabashi-ku, Tokyo 173-8610, Japan E-mail: kkawana-tky@umin.org

Key words: cancer stem cells, spheroid, focal adhesion kinase, glutamine metabolism, mammalian target of rapamycin effects of using FAK inhibitors in ovarian clear cell carcinomas and suggested that targeting glutamine metabolism could overcome the limitation of FAK inhibitors by additively inhibiting the mTOR pathway.

\section{Introduction}

Ovarian cancer has a high mortality rate worldwide, which is related to the late identification of most ovarian cancer cases in advanced stages (1). Late diagnosis indicates that cancer cells are disseminated to intraperitoneal tissues or metastasized to other organs when the cancer is first identified. In these cases, chemotherapy is less likely to be effective, with tumor relapse occurring often, resulting in poor patient prognosis. Developing therapies that improve patient outcomes is an important area of focus $(1,2)$.

The experimental procedure that most appropriately mimic the clinical characteristics of metastasized cancers in vitro is culturing cancer cells in suspension, also known as three-dimensional cultures (3-9). The procedure results in the formation of spheroids, and these spheroids are often considered to share cancer stem-like characteristics (3,10-12).

Accordingly, we have recently obtained spheroids by culturing OVTOKO (ovarian clear cell carcinoma) and $\mathrm{SiHa}$ (cervical squamous cell carcinoma) and demonstrated the common metabolism in spheroids (10). In the present study, by performing RNA-seq analysis and using the Kyoto Encyclopedia of Genes and Genomes (KEGG), we found that the only common trait between spheroids derived from OVTOKO and SiHa was the pathway hsp 04510, which is also known as the focal adhesion kinase (FAK) pathway.

The FAK pathway has been investigated in various types of solid tumors for the last few decades, and this pathway is essential in tumor cell-extracellular matrix attachment, migration, invasion and spheroid formation (13-26). Inhibiting the FAK pathway is promising as a target in ovarian serous 
carcinoma, and currently several types of inhibitors are in clinical trials (27).

However, to the best of our knowledge, there is no study reported on the effectiveness of FAK inhibitors in ovarian clear cell carcinomas. Therefore, we evaluated the effectiveness of targeting the FAK pathway in ovarian clear cell carcinomas using the ovarian clear cell carcinoma cell lines OVTOKO, OVISE and JHOC5. At the same time, we found a potential limitation to inhibiting FAK pathways from the in vitro study: because the phosphorylation of FAK was higher in spheroids than adherent cells, the same doses of FAK inhibitors were not always effective at inhibiting phosphorylation because inhibition depended on cancer status and other conditions.

Thus, we investigated other pathways to overcome the limitation. We have recently demonstrated that the concentration of glutamine and glutamate were significantly higher in spheroids than adherent cells (10), and we investigated the effects of targeting glutamine metabolism in spheroids, especially focusing on the mTOR pathway, which is a common downstream pathway between glutamine metabolism and FAK pathways $(28,29)$. The combination of AOA, a pan-transaminase inhibitor, and PF 573228, an FAK inhibitor, additively inhibited the mTOR pathway in two of three cell lines.

In the present study, from an in vitro point of view, we proposed a rationale for the positive and negative effects of using FAK inhibitors in ovarian clear cell carcinomas and suggested that targeting glutamine metabolism could overcome the limitation of FAK inhibitors by additively inhibiting the mTOR pathway.

\section{Materials and methods}

Cell line and cell culture. The cancer cell lines OVTOKO and OVISE were obtained from the JCRB Cell Bank (Osaka, Japan), and JHOC5 was obtained from RIKEN Cell Bank (Tsukuba, Japan). They were cultured in RPMI-1640 medium or DMEM (Wako, Japan), supplemented with $10 \%$ fetal bovine serum (FBS; Invitrogen, USA) and $100 \mathrm{U} / \mathrm{ml}$ penicillin $/ 100 \mu \mathrm{g} / \mathrm{ml}$ streptomycin (Wako), and sub-cultured by $0.25 \%$ trypsin/EDTA (Wako) detachment. All cells were grown in a humidified atmosphere at $37^{\circ} \mathrm{C}$ with $5 \% \mathrm{CO}_{2}$.

Suspension (spheroid-forming) culture. Dissociated single cells $\left(1 \times 10^{5}\right.$ cells $\left./ \mathrm{ml}\right)$ were seeded into ultra-low attachment plates (Corning Inc., Corning, NY, USA) and cultured for $48 \mathrm{~h}$ unless otherwise described. To collect spheroids, the medium was centrifuged for $2 \mathrm{~min}$ at $100 \mathrm{x} \mathrm{g}$, and the supernatants were carefully aspirated.

mRNA-seq data. Adherent-cultured cells and spheroids were collected after washing and immediately treated with RNAlater (Thermo Fisher Scientific, Waltham, MA, USA). To construct cDNA libraries with the TruSeq RNA library kit, $1 \mu \mathrm{g}$ of total RNA was used. The protocol consisted of polyA-selected RNA extraction, RNA fragmentation, random hexamer primed reverse transcription and 100 nucleotide paired-end sequencing by Illumina HiSeq2000 (Illumina, Inc., San Diego, CA, USA). The libraries were quantified using qPCR according to the qPCR Quantification Protocol Guide and qualified using an Agilent Technologies 2100 Bioanalyzer
(Agilent Technologies, Santa Clara, CA, USA). We processed reads from the sequencer and aligned them to Homo sapiens (hg19) using Tophat v2.0.13 (30). Tophat incorporates the Bowtie v2.2.3 algorithm to perform the alignment (31). Tophat initially removes a portion of the reads based on the quality information accompanying each read before mapping reads to the reference genome. The reference genome sequence of Homo sapiens (hg19) and annotation data were downloaded from the UCSC table browser (http://genome.uscs.edu). Gene annotation information was also used to run Tophat with the '-G' option.

For other parameters in Tophat, the default options were used. Tophat enables multiple alignments per read (up to 20 by default) and a maximum of two mismatches when mapping the reads to the reference. Transcript assembly and abundance estimation were performed using Cufflinks (32). After aligning reads to the genome, Cufflinks v2.2.1 was used to assemble aligned reads into transcripts and estimate the abundance of the reads. To correct sequence expression count bias, '--max-bundle-frags 50000000' options were used. We also used the '-G' option to make the best use of known gene annotation information. For other parameters, default options were used. The transcript counts in the isoform level were calculated, and the relative transcript abundances were measured in FPKM (fragments per kilobase of exon per million fragments mapped) from Cufflinks. Gene level expression values were also calculated from the transcript counts. These values were used for later DEG analysis.

mRNA expression. Raw data were calculated as FPKM of each gene for each sample by Cufflinks software. We excluded genes with zero FPKM values for more than one total sample. We added a filtered gene with an FPKM value to facilitate $\log 10$ transformation. Filtered data was transformed by logarithm and normalized by the quantile normalization method. We finally determined the significant result by adjusting Ifold changel $\geq 2$.

Pathway analysis. Pathway analysis was performed as previously described (33). The Kyoto Encyclopedia of Genes and Genomes (KEGG) database was used to analyze the biological pathways (http://www.genome.jp/kegg/). All data analyses and visualization of differentially expressed genes was conducted using R 3.2.2 (www.r-project.org).

Reagent. L-glutamine ( $2 \mathrm{mM})$ and HEPES were purchased from Wako. PF 573228 (1 $\mu \mathrm{M}$ otherwise described), AOA (2 $\mathrm{mM})$ and dimethyl 2-oxoglutarate $(2 \mathrm{mM})$ were purchased from Sigma-Aldrich (St. Louis, MO, USA).

Western blotting. The same amount of protein from whole cell lysates was subjected to SDS-polyacrylamide gel (Bio-Rad, Hercules, CA, USA) electrophoresis and then electrotransferred onto polyvinylidene difluoride membranes (Millipore, Bedford, MA, USA). Membranes were blocked with 5\% (w/v) skim milk in TBS/Tween-20 for $1 \mathrm{~h}$ at room temperature. After that, the blots were probed with primary antibodies at 1-500 dilutions overnight at $4^{\circ} \mathrm{C}$, followed by incubation with appropriate secondary antibodies conjugated to horseradish peroxidase (GE Healthcare, Tokyo, Japan) for $1 \mathrm{~h}$ at room 
KEGG_PATHWAY (Pathways)

Top 10 Terms of DAVID Functional Annotation Chart
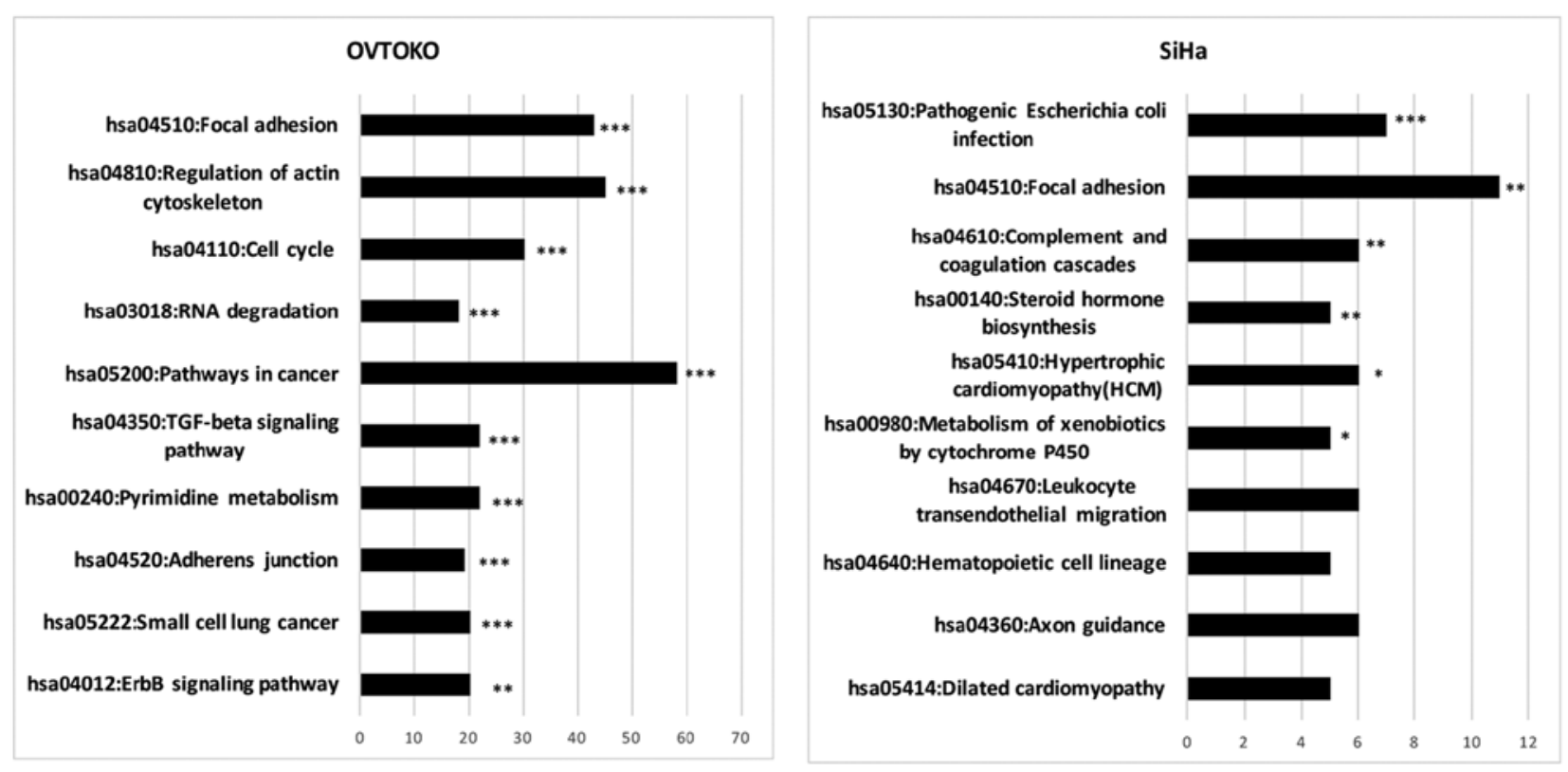

B OVTOKO OVISE JHOC5

C
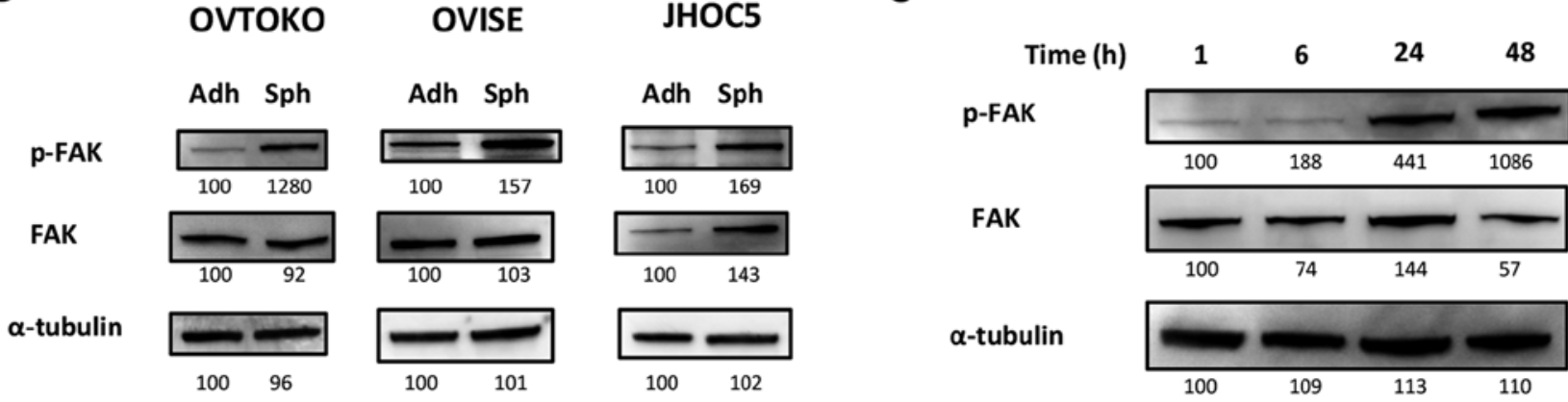

Figure 1. Significance of FAK in spheroids. (A) Result of RNA-seq comparing spheroids and adherent cells. The top 10 pathways based on KEGG are shown. Hsa 04510, the focal adhesion pathway, is the only common pathway between OVTOKO and SiHa. (B) Phosphorylation of FAK in ovarian clear cell carcinoma by western blotting. The phosphorylation level of FAK was higher in spheroids compared to adherent cells. (C) Difference of phosphorylation levels of FAK in different time-course in OVTOKO cells in suspension by western blotting. Cells were seeded in the suspension and then collected at the indicated time after seeding. It was suggested that the increase of the FAK phosphorylation levels of cells in suspension was time-dependent. KEGG, Kyoto Encyclopedia of Genes and Genomes; Adh, adherent-cultured cells; Sph, spheroids. ${ }^{*} \mathrm{P}<0.05 ;{ }^{* *} \mathrm{P}<0.01 ;{ }^{* * *} \mathrm{P}<0.001$.

temperature. The secondary antibodies were detected using Immobilon Western Chemiluminescent HRP Substrate (Millipore) according to the manufacturer's instructions. Protein bands from western blotting were relatively quantified with Image J $(34,35)$.

Antibody. Anti-GLS antibody, anti-GDH antibody, anti-GOT1 antibody and anti-GPT2 antibody were purchased from abcam (cat\# 156876, 89967, 170950 and 101876, respectively).

Anti-PSAT1 antibody was purchased from Proteintech (cat\# 10501-1-AP). Anti-p-FAK (Tyr397) antibody was purchased from Life Technologies (cat\# 44-624G). Anti-FAK antibody was purchased from BD Biosciences (Bedford, MA, USA) (cat\# 610088). Anti-p-Akt (Ser 473) antibody, anti-Akt antibody, anti-p-S6K (Thr389) antibody, anti-S6K antibody, anti-p-S6 (Ser 235/236) antibody and anti-S6 antibody were purchased from Cell signaling technology (cat\# 9271, 9272,
9205, 9202, 4858 and 2217, respectively). Anti- $\alpha$-tubulin antibody was purchased from Millipore (cat\# CP06).

Statistical analysis. P-values $<0.05$ were considered to indicate statistically significant differences.

\section{Results}

Focal adhesion pathway is the only common pathway based on KEGG analysis between spheroids from OVTOKO and SiHa by RNA-seq analysis. We recently obtained spheroids from OVTOKO (ovarian clear cell carcinoma) and SiHa (cervical squamous cell carcinoma) (10). Using these spheroids, we investigated the common pathways, which could be universal and essential pathways in spheroids. The top 10 significant pathways in spheroids compared with adherent-cultured cells from each cell line are shown in Fig. 1. To our surprise, there 
A

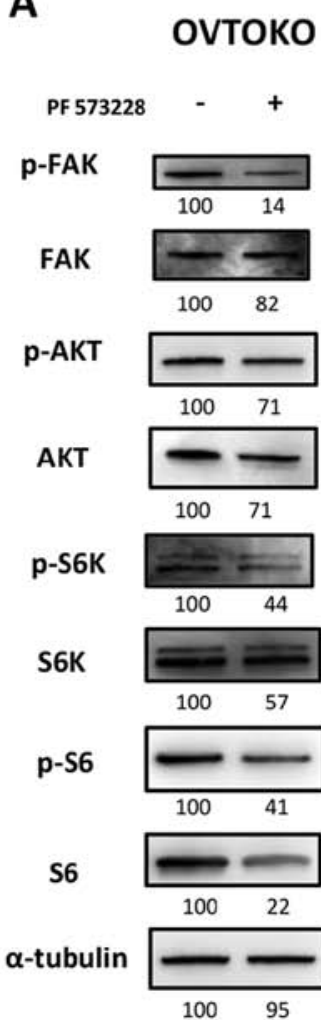

OVISE

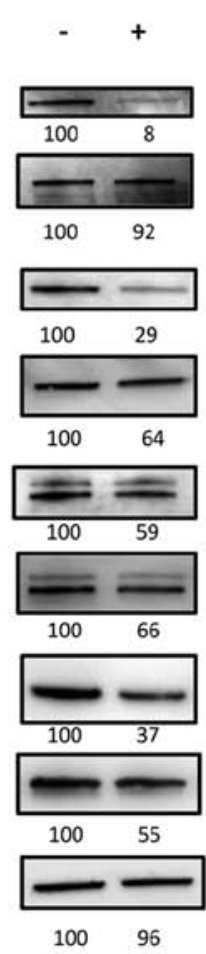

B

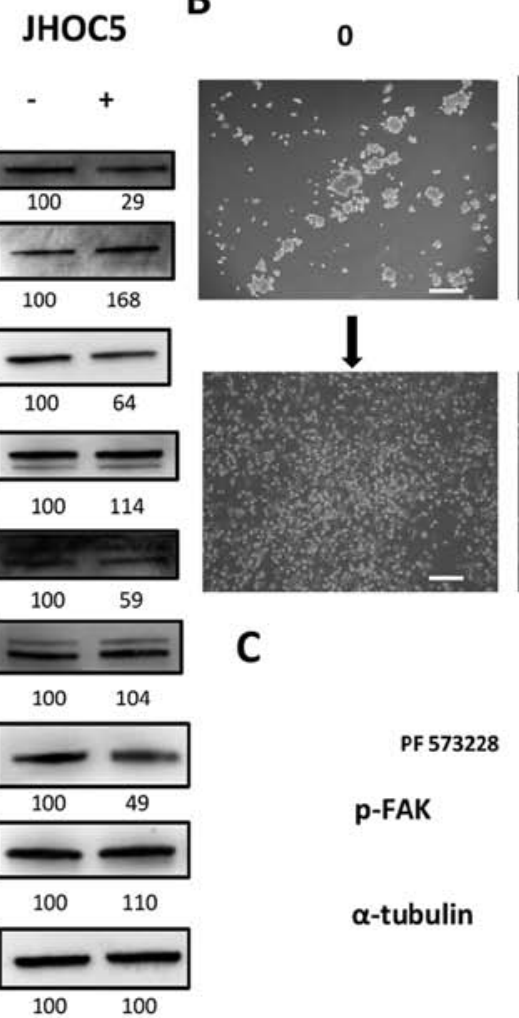

PF $573228(\mu \mathrm{M})$

1
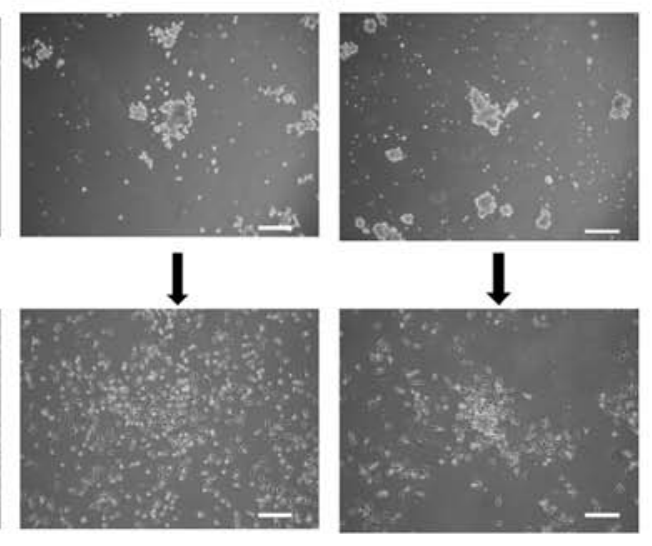

5
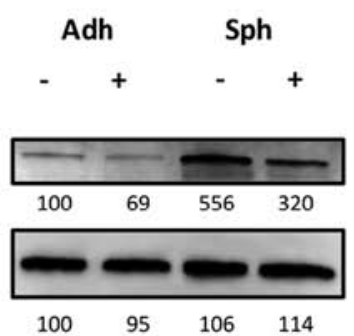

Figure 2. Effect of an FAK inhibitor, PF 573228, on cells in suspension. (A) Inhibitory effect of PF 573228 on FAK/Akt/mTOR pathway. Cells were seeded in the suspension and then cultured with or without $1 \mu \mathrm{M}$ PF 573228. The phosphorylation of FAK and the Akt/mTOR pathway were inhibited. (B) Effect of PF 573228 on spheroid formation and expansion in adherent plates. Cells were seeded in the suspension and then cultured for $48 \mathrm{~h}$ with or without PF 573228. Then, spheroids were collected and reseeded into adherent plates. The medium was refreshed $1 \mathrm{~h}$ after re-seeding (which resulted in removal of non-attached spheroids). Then, attached spheroids were cultured for another $24 \mathrm{~h}$. It was not obvious that PF 573228 inhibited spheroid formation. However, it appeared that the population of attached spheroids reduced in a dose-dependent manner. Bars, $100 \mu \mathrm{M}$. (C) Differential impact of PF 573228 on OVTOKO cells between adherent cells and spheroids. Cells in adherent plates or in suspension were cultured for $48 \mathrm{~h}$ with or without $1 \mu \mathrm{M}$ PF 573228 . The phosphorylation levels of FAK of cells in suspension could not be reduced to the level of those of adherent cells. Adh, adherent-cultured cells; Sph, spheroids.

was only one pathway that was common between spheroids from OVTOKO and those from $\mathrm{SiHa}$; it was hsa 04510, the focal adhesion pathway.

Phosphorylation of FAK is increased in spheroids compared to adherent cells, and the increase is time-dependent. Inhibition of the FAK pathway is a promising target in ovarian serous carcinoma $(15,25,27,36,37)$, and we focused on confirming the effectiveness of targeting the FAK pathway in ovarian clear cell carcinoma using the ovarian clear cell carcinoma cell lines OVTOKO, OVISE and JHOC5. We investigated the expression levels of FAK and the phosphorylation of FAK using western blots. As shown in Fig. 1B, the phosphorylation of FAK was higher in spheroids than adherent cells, and it was suggested that the increase was time-dependent after being in suspension (Fig. 1C).

PF 573228, a FAK inhibitor, decreases the phosphorylation of FAK and inhibits the downstream pathway, the Akt/mTOR pathway. PF 573228 is a FAK inhibitor, and it was shown to be effective at inhibiting tumor migration in various types of solid tumors by inhibiting the phosphorylation of FAK (27). We obtained the expected results, where PF 573228 decreased the phosphorylation of FAK and inhibited the downstream pathway, the Akt/mTOR pathway (Fig. 2A). Although it was difficult to quantify, it appeared that exposing cells in suspension to PF 573228 did not affect spheroid-forming abilities. However, we found that the attachment and proliferation ability of spheroids cultured with PF 573228 were deterred when reseeded into adherent plates (Fig. 2B).

Sensitivity and impact of FAK pathway inhibition depends on the cancer cell status. The results above were expected based on previous studies and the presence of FAK inhibitors in clinical trials $(27,37-40)$. We also determined that the effectiveness of inhibiting FAK pathways might depend on cancer cell status. The phosphorylation of FAK in spheroids was higher than adherent cells, and exposing them to $1 \mu \mathrm{M}$ PF 573228 still resulted in a higher phosphorylation level of FAK compared with that of the non-exposed adherent cells (Fig. 2C).

Glutamine metabolism is essential to maintain the mTOR pathway of cells in suspension. We recently demonstrated that the concentration of glutamine and glutamate were significantly increased in spheroids compared to adherent cells (10), and we investigated the effects of targeting glutamine metabolism on spheroids, especially focusing on the mTOR pathway. This was our focus because it is a common downstream pathway between glutamine metabolism and 
A

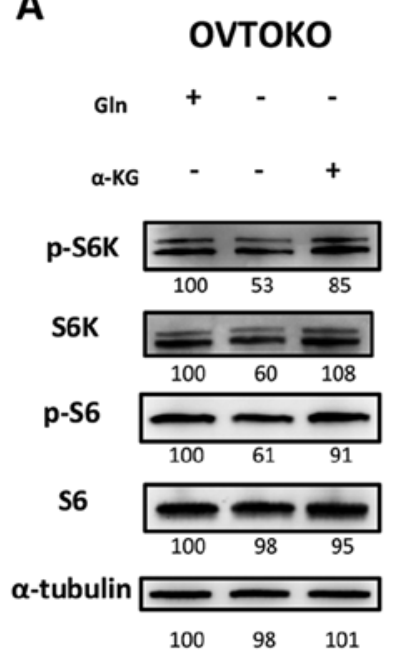

OVISE

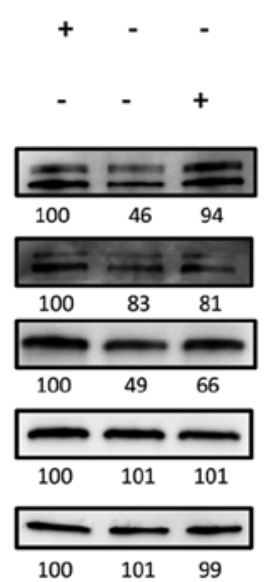

B

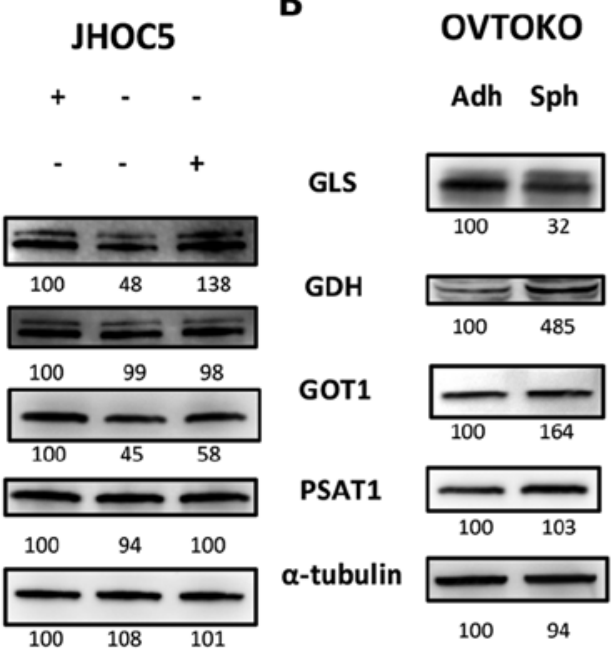

OVISE

Adh Sph

JHOC5

Adh Sph

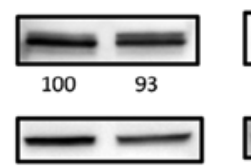

$100 \quad 27$
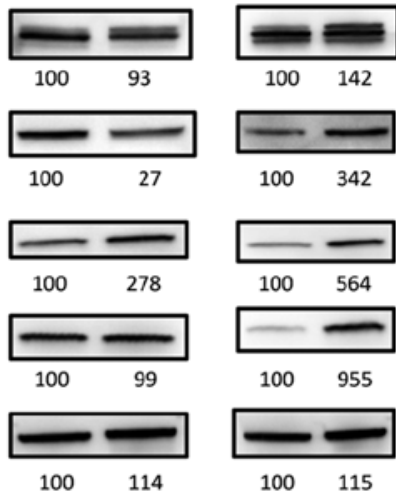

$100 \quad 342$

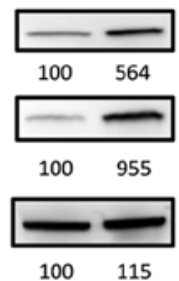

C оVтоко
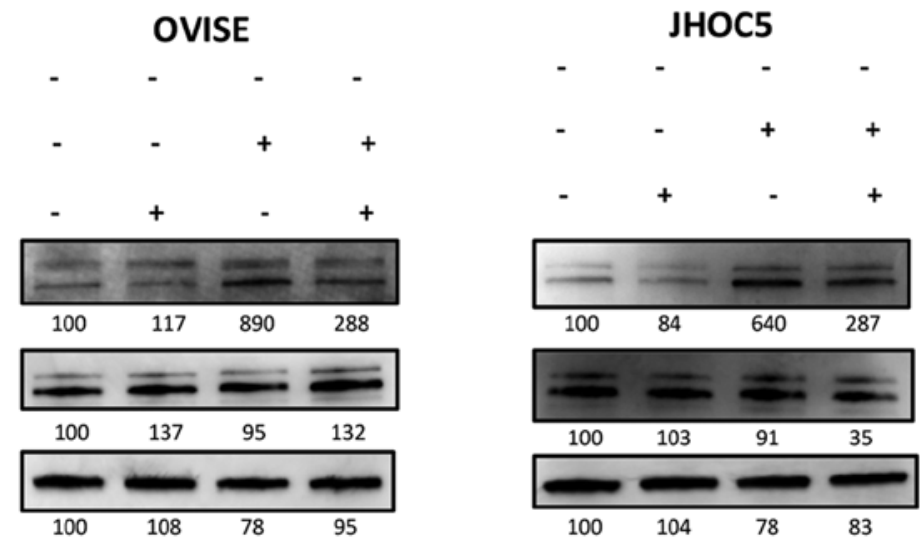

Figure 3. Significance of glutamine metabolism in cells in suspension. (A) Glutamine metabolism impact on the mTOR pathway in cells in suspension. Cells were cultured for $6 \mathrm{~h}$ with or without $2 \mathrm{mM}$ glutamine and $2 \mathrm{mM}$ dimethyl 2-oxoglutarate. Glutamine depletion inhibited the mTOR pathway and dimethyl 2-oxoglutarate salvaged it. This suggested that glutamine metabolism had some impacts on the mTOR pathway of cells in suspension. (B) Expression of enzymes related to glutamine metabolism between adherent cells and spheroids. Expression of enzymes related during glutamine metabolism (GLS, GDH, GOT1, PSAT1 and GPT2) were investigated by western blotting. Only GOT1 was commonly increased in spheroids compared to adherent cells among all cell lines. The expression of GPT2 could not be confirmed. (C) The effect of AOA, a pan-transaminase inhibitor, on the mTOR pathway related to glutamine metabolism for cells in suspension. Cells in suspension were cultured for $6 \mathrm{~h}$ with or without $2 \mathrm{mM}$ dimethyl 2-oxoglutarate and $2 \mathrm{mM}$ AOA. The salvation effect of dimethyl 2-oxoglutarate on the mTOR pathway inhibited by glutamine depletion was cancelled by AOA. $\alpha$-KG, dimethyl 2-oxoglutarate; GLS, glutaminase; GDH, glutamate dehydrogenase; GOT, aspartate aminotransferase; PSAT1, phosphohydroxythreonine aminotransferase; GPT2, alanine aminotransferase; Adh, adherent-cultured cells; and Sph, spheroids.

FAK pathways and because inhibiting the mTOR pathway is a promising target for the treatment for ovarian clear cell carcinoma $(28,29,36,41,42)$. To confirm the significance of glutamine metabolism in cells in suspension, we cultured cells in suspension with or without glutamine. We found that the mTOR pathway, which is represented by the phosphorylation levels of S6K and S6, was inhibited when cells were cultured without glutamine. We also found that the effect was salvaged by supplementation of cell-permeable type $\alpha-K G$, which are the intermediates of both glutaminolysis and the tricarboxylic acid (TCA) cycle (Fig. 3A) (43-45). This result suggested the involvement of glutaminolysis in maintaining the mTOR pathway. We then investigated major enzymes related to glutamine metabolism, including glutaminase (GLS), glutamate dehydrogenase (GDH), aspartate aminotransferase (GOT1), phosphohydroxythreonine aminotransferase (PSAT1) and alanine aminotransferase (GPT2) $(46,47)$. We could not detect GPT2 (data not shown). We found that the expression of GOT1 was commonly increased in spheroids compared to adherent cells among the cell lines (Fig. 3B). Amino-oxyacetate (AOA), a pan-transaminase inhibitor (48-50), cancelled the salvation effect of $\alpha-K G$ (Fig. 3C) on the mTOR pathway when inhibited by glutamine depletion.

Targeting glutamine metabolism additively inhibited the mTOR pathway of spheroids when combined with a FAK inhibitor. We then investigated the synergistic effect of AOA combined with a FAK inhibitor on the mTOR pathway in ovarian clear cell carcinoma. We found that exposing cells in suspension to AOA and PF 573228 additively inhibited the mTOR pathway in OVISE and JHOC5 (Fig. 4). The combined effect could not be observed in spheroids from OVTOKO. Rather, to our surprise, it appeared that adding AOA cancelled the inhibitory effect of PF 573228 on the mTOR pathway (Fig. 4). We were unsure what caused the adverse effect of AOA in spheroids from OVTOKO, which is discussed later. 
оVтоко

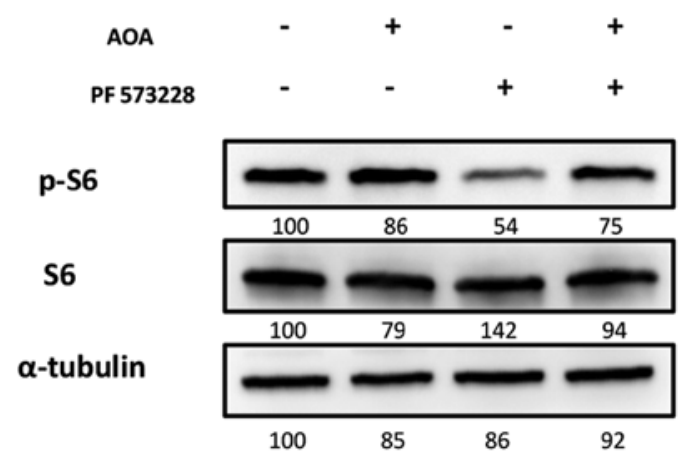

OVISE

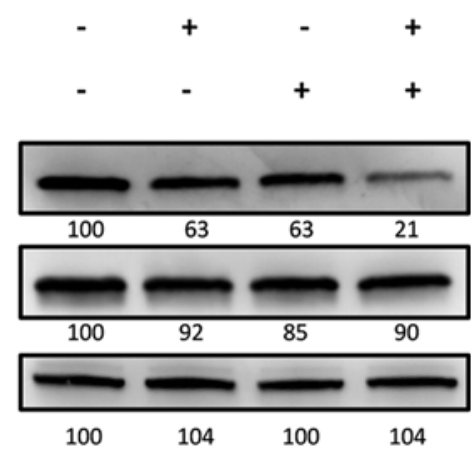

JHOC5

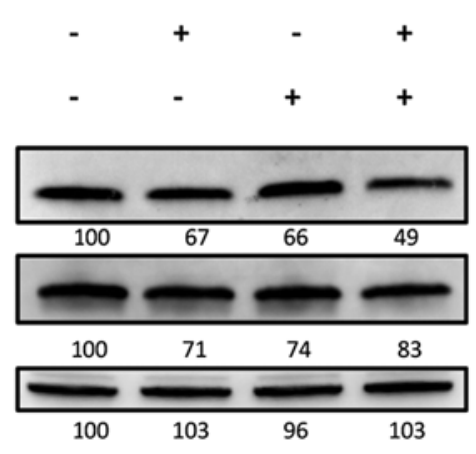

Figure 4. Synergistic effect of targeting glutamine metabolism and FAK on the mTOR pathway. Synergistic effect of AOA and PF 573228 on mTOR pathway in cells in suspension. Cells in the suspension were cultured for $48 \mathrm{~h}$ with or without $2 \mathrm{mM}$ AOA and PF 573228. The phosphorylation level of S6 was additively reduced by AOA and PF 573228 treatment in OVISE and JHOC5. The combined effect could not be observed in OVTOKO. Rather, it appeared that adding AOA cancelled the inhibitory effect of PF 573228 on the mTOR pathway.

\section{Discussion}

We examined the positive and negative effects of targeting the FAK pathway in ovarian clear cell carcinoma and suggested that targeting glutamine metabolism might overcome the limitation of inhibiting the FAK pathway by additively inhibiting the mTOR pathway of cancer stem-like properties.

Ovarian cancer is one of the leading cause of death in the world. Fatality results because it seldom induces symptoms and is often identified during advanced stages (1). In these cases, chemotherapy is less likely to be effective, and it often results in tumor relapse. The experimental procedures that reflect the clinical characteristics of metastasized cancers in vitro are culturing cancer cells in suspension, which results in the formation of spheroids $(3,4)$.

Accordingly, we performed RNA-seq analysis in spheroids obtained from OVTOKO (ovarian clear cell carcinoma) and $\mathrm{SiHa}$ (cervical squamous cell carcinoma) to find targets that were universal and essential pathways in spheroids. We found that the only common pathway based on KEGG was hsp 04510, the focal adhesion pathway. The focal adhesion pathway has been investigated in various types of solid tumors for the last few decades, and inhibition of FAK is a promising target in ovarian serous adenocarcinoma $(15,25,27,36,37)$. Thus, we evaluated the effectiveness of targeting the FAK pathway in ovarian clear cell carcinoma using the ovarian clear cell carcinoma cell lines OVTOKO, OVISE and JHOC5.

In the first half of this study, we demonstrated that the phosphorylation of FAK was increased in spheroids compared to adherent cells, and inhibiting FAK phosphorylation resulted in deterring spheroid attachment onto adherent plates. These findings suggested that targeting the FAK pathway can be effective in targeting cancer stem cells because spheroids are known to share cancer stem-like features (positive effect) $(3,10-12)$. We also demonstrated the limitation of targeting the FAK pathway from an in vitro study (negative effect). The phosphorylation levels of FAK in spheroids were high, and even as much as $1 \mu \mathrm{M}$ PF 573228 could not completely inhibit the phosphorylation of FAK. These results intrigued us to determine a way to overcome it. In the latter half of this study, we approached FAK and the downstream mTOR pathway from a glutamine metabolism perspective.

We recently demonstrated that the concentrations of glutamine and glutamate were significantly increased in spheroids compared to adherent cells (10). Glutamine metabolism is known to be essential in the mTOR pathway (28). Taken together, we speculated that targeting glutamine metabolism could be a breakthrough for the potential limitation of targeting FAK in ovarian clear cell carcinoma. Among enzymes related to glutamine metabolism, only the expression of GOT1 was commonly increased in spheroids compared to adherent cells among all cell lines. The combination of AOA, a pan-transaminase inhibitor, and PF 573228, an FAK inhibitor, additively inhibited the mTOR pathway in two of three cell lines.

The combined effect was not observed in spheroids from OVTOKO. Alternatively, it seemed that adding AOA cancelled the inhibitory effect of PF 573228 on the mTOR pathway. We are not sure what caused the adverse effect of AOA in spheroids from OVTOKO. Previous studies demonstrated that the KRAS mutation caused a metabolic switch and changed the significance of GOT1 in glutaminolysis in pancreatic cancer (50). However, it seemed that the KRAS mutation was not the one that caused our results, based on our previous study that investigated the mutation of each oncogene in ovarian clear cell carcinoma cell lines (51). Finding a biomarker and features to predict the combinational effect of targeting glutamine metabolism and FAK will be our future direction.

In conclusion, from the in vitro perspective, we proposed that targeting glutamine metabolism with properly chosen targets could enhance the inhibitory effect of FAK inhibitors on the mTOR pathway in cancer stem cell-like properties.

\section{Acknowledgements}

This work was supported by JSPS KAKENHI grant number 26293357 and 15H06172, and by the Practical Research for Innovative Cancer Control from Japan Agency for Medical Research and Development, AMED. 


\section{References}

1. Agarwal R and Kaye SB: Ovarian cancer: Strategies for overcoming resistance to chemotherapy. Nat Rev Cancer 3: 502-516, 2003 .

2. Tancioni I, Uryu S, Sulzmaier FJ, Shah NR, Lawson C, Miller NL, Jean C, Chen XL, Ward KK and Schlaepfer DD: FAK Inhibition disrupts a $\beta 5$ integrin signaling axis controlling anchorage-independent ovarian carcinoma growth. Mol Cancer Ther 13: 2050-2061, 2014.

3. Friedrich J, Ebner R and Kunz-Schughart LA: Experimental anti-tumor therapy in 3-D: Spheroids - old hat or new challenge? Int J Radiat Biol 83: 849-871, 2007.

4. Friedrich J, Seidel C, Ebner R and Kunz-Schughart LA Spheroid-based drug screen: Considerations and practical approach. Nat Protoc 4: 309-324, 2009.

5. Beck HC, Gosau M, Kristensen LP and Morsczeck C: A sitespecific phosphorylation of the focal adhesion kinase controls the formation of spheroid cell clusters. Neurochem Res 39 $1199-1205,2014$

6. Heyman L, Kellouche S, Fernandes J, Dutoit S, Poulain L and Carreiras F: Vitronectin and its receptors partly mediate adhesion of ovarian cancer cells to peritoneal mesothelium in vitro. Tumour Biol 29: 231-244, 2008.

7. Tanjoni I, Walsh C, Uryu S, Tomar A, Nam JO, Mielgo A, Lim ST, Liang C, Koenig M, Sun C, et al: PND-1186 FAK inhibitor selectively promotes tumor cell apoptosis in three-dimensional environments. Cancer Biol Ther 9: 764-777, 2010.

8. Ward KK, Tancioni I, Lawson C, Miller NL, Jean C, Chen XL, Uryu S, Kim J, Tarin D, Stupack DG, et al: Inhibition of focal adhesion kinase (FAK) activity prevents anchorage-independent ovarian carcinoma cell growth and tumor progression. Clin Exp Metastasis 30: 579-594, 2012.

9. Tancioni I, Miller NL, Uryu S, Lawson C, Jean C, Chen XL, Kleinschmidt EG and Schlaepfer DD: FAK activity protects nucleostemin in facilitating breast cancer spheroid and tumor growth. Breast Cancer Res 17: 47, 2015.

10. Sato M, Kawana K, Adachi K, Fujimoto A, Yoshida M, Nakamura H, Nishida H, Inoue T, Taguchi A, Takahashi J, et al: Spheroid cancer stem cells display reprogrammed metabolism and obtain energy by actively running the tricarboxylic acid (TCA) cycle. Oncotarget 7: 33297-33305, 2016.

11. Shield K, Ackland ML, Ahmed N and Rice GE: Multicellular spheroids in ovarian cancer metastases: Biology and pathology. Gynecol Oncol 113: 143-148, 2009.

12. Visvader JE and Lindeman GJ: Cancer stem cells in solid tumours: Accumulating evidence and unresolved questions. Nat Rev Cancer 8: 755-768, 2008

13. Alanko J, Mai A, Jacquemet G, Schauer K, Kaukonen R, Saari M, Goud B and Ivaska J: Integrin endosomal signalling suppresses anoikis. Nat Cell Biol 17: 1412-1421, 2015.

14. Lark AL, Livasy CA, Calvo B, Caskey L, Moore DT, Yang X and Cance WG: Overexpression of focal adhesion kinase in primary colorectal carcinomas and colorectal liver metastases: immunohistochemistry and real-time PCR analyses. Clin Cancer Res 9: 215-222, 2003

15. Sood AK, Coffin JE, Schneider GB, Fletcher MS, DeYoung BR, Gruman LM, Gershenson DM, Schaller MD and Hendrix MJ: Biological significance of focal adhesion kinase in ovarian cancer: Role in migration and invasion. Am J Pathol 165 1087-1095, 2004.

16. Beierle EA, Massoll NA, Hartwich J, Kurenova EV, Golubovskaya VM, Cance WG, McGrady P and London WB: Focal adhesion kinase expression in human neuroblastoma: Immunohistochemical and real-time PCR analyses. Clin Cancer Res 14: 3299-3305, 2008.

17. Casanova I, Parreño M, Farré L, Guerrero S, Céspedes MV, Pavon MA, Sancho FJ, Marcuello E, Trias M and Mangues R: Celecoxib induces anoikis in human colon carcinoma cells associated with the deregulation of focal adhesions and nuclear translocation of p130Cas. Int J Cancer 118: 2381-2389, 2006.

18. Chen CH, Shyu MK, Wang SW, Chou CH, Huang MJ, Lin TC, Chen ST, Lin HH and Huang MC: MUC20 promotes aggressive phenotypes of epithelial ovarian cancer cells via activation of the integrin $\beta 1$ pathway. Gynecol Oncol 140: 131-137, 2016.

19. Chen YY, Wang ZX, Chang PA, Li JJ, Pan F, Yang L, Bian ZH, Zou L, He JM and Liang HJ: Knockdown of focal adhesion kinase reverses colon carcinoma multicellular resistance. Cancer Sci 100: 1708-1713, 2009.
20. Kong D, Chen F and Sima NI: Inhibition of focal adhesion kinase induces apoptosis in bladder cancer cells via Src and the phosphatidylinositol 3-kinase/Akt pathway. Exp Ther Med 10: $1725-1731,2015$

21. Lark AL, Livasy CA, Dressler L, Moore DT, Millikan RC, Geradts J, Iacocca M, Cowan D, Little D, Craven RJ, et al: High focal adhesion kinase expression in invasive breast carcinomas is associated with an aggressive phenotype. Mod Pathol 18: 1289-1294, 2005

22. Liu W, Bloom DA, Cance WG, Kurenova EV, Golubovskay VM and Hochwald SN: FAK and IGF-IR interact to provide survival signals in human pancreatic adenocarcinoma cells. Carcinogenesis 29: 1096-1107, 2008.

23. Madan R, Smolkin MB, Cocker R, Fayyad R and Oktay MH: Focal adhesion proteins as markers of malignant transformation and prognostic indicators in breast carcinoma. Hum Pathol 37: 9-15, 2006

24. Rentala S, Chintala R, Guda M, Chintala M, Komarraju AL and Mangamoori LN: Atorvastatin inhibited Rho-associated kinase 1 (ROCK1) and focal adhesion kinase (FAK) mediated adhesion and differentiation of $\mathrm{CD} 133{ }^{+} \mathrm{CD} 44^{+}$prostate cancer stem cells. Biochem Biophys Res Commun 441: 586-592, 2013.

25. Stone RL, Baggerly KA, Armaiz-Pena GN, Kang Y, Sanguino AM, Thanapprapasr D, Dalton HJ, Bottsford-Miller J, Zand B, Akbani R, et al: Focal adhesion kinase: An alternative focus for anti-angiogenesis therapy in ovarian cancer. Cancer Biol Ther 15: 919-929, 2014.

26. Sulzmaier FJ, Jean C and Schlaepfer DD: FAK in cancer: Mechanistic findings and clinical applications. Nat Rev Cancer 14: 598-610, 2014.

27. Lee BY, Timpson P, Horvath LG and Daly RJ: FAK signaling in human cancer as a target for therapeutics. Pharmacol Ther 146: 132-149, 2015.

28. Durán RV, Oppliger W, Robitaille AM, Heiserich L, Skendaj R, Gottlieb E and Hall MN: Glutaminolysis activates Rag-mTORC1 signaling. Mol Cell 47: 349-358, 2012.

29. Kassem L and Abdel-Rahman O: Targeting mTOR pathway in gynecological malignancies: Biological rationale and systematic review of published data. Crit Rev Oncol Hematol 108: 1-12, 2016.

30. Trapnell C, Pachter L and Salzberg SL: TopHat: Discovering splice junctions with RNA-Seq. Bioinformatics 25: 1105-1111, 2009.

31. Langmead B, Trapnell C, Pop M and Salzberg SL: Ultrafast and memory-efficient alignment of short DNA sequences to the human genome. Genome Biol 10: R25, 2009.

32. Trapnell C, Williams BA, Pertea G, Mortazavi A, Kwan G, van Baren MJ, Salzberg SL, Wold BJ and Pachter L: Transcript assembly and quantification by RNA-Seq reveals unannotated transcripts and isoform switching during cell differentiation. Nat Biotechnol 28: 511-515, 2010.

33. Zhang Y, He RQ, Dang YW, Zhang XL, Wang X, Huang SN, Huang WT, Jiang MT, Gan XN, Xie Y, et al: Comprehensive analysis of the long noncoding RNA HOXA11-AS gene interaction regulatory network in NSCLC cells. Cancer Cell Int 16 89, 2016.

34. Fujimoto A, Kawana K, Taguchi A, Adachi K, Sato M, Nakamura H, Ogishima J, Yoshida M, Inoue T, Nishida H, et al: Inhibition of endoplasmic reticulum (ER) stress sensors sensitizes cancer stem-like cells to ER stress-mediated apoptosis. Oncotarget 7: 51854-51864, 2016.

35. Schneider CA, Rasband WS and Eliceiri KW: NIH Image to ImageJ: 25 years of image analysis. Nat Methods 9: 671-675, 2012.

36. Kang Y, Hu W, Ivan C, Dalton HJ, Miyake T, Pecot CV, Zand B, Liu T, Huang J, Jennings NB, et al: Role of focal adhesion kinase in regulating YB-1-mediated paclitaxel resistance in ovarian cancer. J Natl Cancer Inst 105: 1485-1495, 2013.

37. McGrail DJ, Khambhati NN, Qi MX, Patel KS, Ravikumar N, Brandenburg CP and Dawson MR: Alterations in ovarian cancer cell adhesion drive taxol resistance by increasing microtubule dynamics in a FAK-dependent manner. Sci Rep 5: 9529, 2015.

38. Infante JR, Camidge DR, Mileshkin LR, Chen EX, Hicks RJ, Rischin D, Fingert H, Pierce KJ, Xu H, Roberts WG, et al: Safety, pharmacokinetic, and pharmacodynamic phase I dose-escalation trial of PF-00562271, an inhibitor of focal adhesion kinase, in advanced solid tumors. J Clin Oncol 30: 1527-1533, 2012.

39. Schultze A and Fiedler W: Therapeutic potential and limitations of new FAK inhibitors in the treatment of cancer. Expert Opin Investig Drugs 19: 777-788, 2010. 
40. Walsh C, Tanjoni I, Uryu S, Tomar A, Nam J-O, Luo H, Phillips A Patel N, Kwok C, McMahon G, et al: Oral delivery of PND-1186 FAK inhibitor decreases tumor growth and spontaneous breast to lung metastasis in pre-clinical models. Cancer Biol Ther 9: 778-790, 2010 .

41. Mabuchi S, Kuroda H, Takahashi R and Sasano T: The PI3K/ AKT/mTOR pathway as a therapeutic target in ovarian cancer. Gynecol Oncol 137: 173-179, 2015.

42. Yamamoto D, Sonoda Y, Hasegawa M, Funakoshi-Tago M, Aizu-Yokota E and Kasahara T: FAK overexpression upregulates cyclin D3 and enhances cell proliferation via the PKC and PI3-kinase-Akt pathways. Cell Signal 15: 575-583, 2003.

43. Hou P, Kuo CY, Cheng CT, Liou JP, Ann DK and Chen Q: Intermediary metabolite precursor dimethyl-2-ketoglutarate stabilizes hypoxia-inducible factor- $1 \alpha$ by inhibiting prolyl-4hydroxylase PHD2. PLoS One 9: e113865, 2014.

44. Zhao J, Peng L, Luo Z, Cui R and Yan M: Inhibitory effects of dimethyl $\alpha$-ketoglutarate in hepatic stellate cell activation. Int J Clin Exp Pathol 8: 5471-5477, 2015.

45. Mariño G, Pietrocola F, Kong Y, Eisenberg T, Hill JA, Madeo F and Kroemer G: Dimethyl $\alpha$-ketoglutarate inhibits maladaptive autophagy in pressure overload-induced cardiomyopathy. Autophagy 10: 930-932, 2014.
46. Jin L, Alesi GN and Kang S: Glutaminolysis as a target for cancer therapy. Oncogene 35: 3619-3625, 2016.

47. Yang L, Moss T, Mangala LS, Marini J, Zhao H, Wahlig S, Armaiz-Pena G, Jiang D, Achreja A, Win J, et al: Metabolic shifts toward glutamine regulate tumor growth, invasion and bioenergetics in ovarian cancer. Mol Syst Biol 10: 728, 2014

48. Feld FM, Nagel PD, Weissinger SE, Welke C, Stenzinger A Möller P and Lennerz JK: GOT1/AST1 expression status as a prognostic biomarker in pancreatic ductal adenocarcinoma. Oncotarget 6: 4516-4526, 2015.

49. Thornburg JM, Nelson KK, Clem BF, Lane AN, Arumugam S, Simmons A, Eaton JW, Telang S and Chesney J: Targeting aspartate aminotransferase in breast cancer. Breast Cancer Res 10: R84, 2008

50. Son J, Lyssiotis CA, Ying H, Wang X, Hua S, Ligorio M, Perera RM, Ferrone CR, Mullarky E, Shyh-Chang N, et al: Glutamine supports pancreatic cancer growth through a KRASregulated metabolic pathway. Nature 496: 101-105, 2013.

51. Kashiyama T, Oda K, Ikeda Y, Shiose Y, Hirota Y, Inaba K, Makii C, Kurikawa R, Miyasaka A, Koso T, et al: Antitumor activity and induction of TP53-dependent apoptosis toward ovarian clear cell adenocarcinoma by the dual PI3K/mTOR inhibitor DS-7423. PLoS One 9: e87220, 2014. 